\title{
Nilpotent Groups
}

\author{
Dailu Li \\ Qingdao University of Science \\ and Technology \\ China
}

\author{
Xiquan Liang \\ Qingdao University of Science \\ and Technology \\ China
}

\author{
Yanhong Men \\ Qingdao University of Science \\ and Technology \\ China
}

Summary. This article describes the concept of the nilpotent group and some properties of the nilpotent groups.

MML identifier: GRNILP_1, version: $\underline{7.11 .04 \quad 4.130 .1076}$

The papers [2], [3], [4], [6], [7], [5], [8], [9], [10], and [1] provide the terminology and notation for this paper.

For simplicity, we adopt the following convention: $x$ denotes a set, $G$ denotes a group, $A, B, H, H_{1}, H_{2}$ denote subgroups of $G, a, b, c$ denote elements of $G, F$ denotes a finite sequence of elements of the carrier of $G$, and $i, j$ denote elements of $\mathbb{N}$.

One can prove the following propositions:

(1) $a^{b}=a \cdot[a, b]$.

(2) $[a, b]^{-1}=\left[a, b^{-1}\right]^{b}$.

(3) $[a, b]^{-1}=\left[a^{-1}, b\right]^{a}$.

(4) $\left(\left[a, b^{-1}\right]^{b}\right)^{-1}=\left[b^{-1}, a\right]^{b}$.

(5) $\left[a, b^{-1}, c\right]^{b}=\left[\left[a, b^{-1}\right]^{b}, c^{b}\right]$.

(6) $\left[a, b^{-1}\right]^{b}=[b, a]$.

(7) $\left[a, b^{-1}, c\right]^{b}=\left[b, a, c^{b}\right]$.

(8) $\left[a, b, c^{a}\right] \cdot\left[c, a, b^{c}\right] \cdot\left[b, c, a^{b}\right]=\mathbf{1}_{G}$. 
(9) $[A, B]$ is a subgroup of $[B, A]$.

(10) $[A, B]=[B, A]$.

Let us consider $G, A, B$. Let us note that the functor $[A, B]$ is commutative.

One can prove the following propositions:

(11) If $B$ is a subgroup of $A$, then the commutators of $A \& B \subseteq \bar{A}$.

(12) If $B$ is a subgroup of $A$, then $[A, B]$ is a subgroup of $A$.

(13) If $B$ is a subgroup of $A$, then $[B, A]$ is a subgroup of $A$.

(14) If $\left[H_{1}, \Omega_{G}\right]$ is a subgroup of $H_{2}$, then $\left[H_{1} \cap H, H\right]$ is a subgroup of $H_{2} \cap H$.

(15) $\left[H_{1}, H_{2}\right]$ is a subgroup of $\left[H_{1}, \Omega_{G}\right]$.

(16) $A$ is a normal subgroup of $G$ iff $\left[A, \Omega_{G}\right]$ is a subgroup of $A$.

Let us consider $G$. The normal subgroups of $G$ yields a set and is defined by:

(Def. 1) $x \in$ the normal subgroups of $G$ iff $x$ is a strict normal subgroup of $G$.

Let us consider $G$. One can verify that the normal subgroups of $G$ is non empty.

Next we state three propositions:

(17) Let $F$ be a finite sequence of elements of the normal subgroups of $G$ and given $j$. If $j \in \operatorname{dom} F$, then $F(j)$ is a strict normal subgroup of $G$.

(18) The normal subgroups of $G \subseteq \operatorname{SubGr} G$.

(19) Every finite sequence of elements of the normal subgroups of $G$ is a finite sequence of elements of SubGr $G$.

Let $I_{1}$ be a group. We say that $I_{1}$ is nilpotent if and only if the condition (Def. 2) is satisfied.

(Def. 2) There exists a finite sequence $F$ of elements of the normal subgroups of $I_{1}$ such that

(i) $\operatorname{len} F>0$,

(ii) $\quad F(1)=\Omega_{\left(I_{1}\right)}$,

(iii) $F($ len $F)=\{\mathbf{1}\}_{\left(I_{1}\right)}$, and

(iv) for every $i$ such that $i, i+1 \in \operatorname{dom} F$ and for all strict normal subgroups $G_{1}, G_{2}$ of $I_{1}$ such that $G_{1}=F(i)$ and $G_{2}=F(i+1)$ holds $G_{2}$ is a subgroup of $G_{1}$ and $G_{1} /\left(G_{2}\right)_{\left(G_{1}\right)}$ is a subgroup of $\mathrm{Z}\left({ }^{I_{1}} / G_{2}\right)$.

Let us note that there exists a group which is nilpotent and strict.

We now state four propositions:

(20) Let $G_{1}$ be a subgroup of $G$ and $N$ be a strict normal subgroup of $G$. Suppose $N$ is a subgroup of $G_{1}$ and $G_{1} /{ }_{(N)_{\left(G_{1}\right)}}$ is a subgroup of $\mathrm{Z}\left({ }^{G} / N\right)$. Then $\left[G_{1}, \Omega_{G}\right]$ is a subgroup of $N$.

(21) Let $G_{1}$ be a subgroup of $G$ and $N$ be a normal subgroup of $G$. Suppose $N$ is a strict subgroup of $G_{1}$ and $\left[G_{1}, \Omega_{G}\right]$ is a strict subgroup of $N$. Then $G_{1} /(N)_{\left(G_{1}\right)}$ is a subgroup of $\mathrm{Z}(G / N)$. 
(22) Let $G$ be a group. Then $G$ is nilpotent if and only if there exists a finite sequence $F$ of elements of the normal subgroups of $G$ such that len $F>0$ and $F(1)=\Omega_{G}$ and $F($ len $F)=\{\mathbf{1}\}_{G}$ and for every $i$ such that $i, i+1 \in \operatorname{dom} F$ and for all strict normal subgroups $G_{1}, G_{2}$ of $G$ such that $G_{1}=F(i)$ and $G_{2}=F(i+1)$ holds $G_{2}$ is a subgroup of $G_{1}$ and $\left[G_{1}, \Omega_{G}\right]$ is a subgroup of $G_{2}$.

(23) Let $G$ be a group, $H, G_{1}$ be subgroups of $G, G_{2}$ be a strict normal subgroup of $G, H_{1}$ be a subgroup of $H$, and $H_{2}$ be a normal subgroup of $H$. Suppose $G_{2}$ is a subgroup of $G_{1}$ and $G_{1} /\left(G_{2}\right)_{\left(G_{1}\right)}$ is a subgroup of $\mathrm{Z}\left({ }^{G} / G_{2}\right)$ and $H_{1}=G_{1} \cap H$ and $H_{2}=G_{2} \cap H$. Then $H_{1} /{\left(H_{2}\right)_{\left(H_{1}\right)}}$ is a subgroup of $\mathrm{Z}\left(\mathrm{H}^{\mathrm{H}} / \mathrm{H}_{2}\right)$.

Let $G$ be a nilpotent group. Note that every subgroup of $G$ is nilpotent.

Let us mention that every group which is commutative is also nilpotent and every group which is cyclic is also nilpotent.

We now state four propositions:

(24) Let $G, H$ be strict groups, $h$ be a homomorphism from $G$ to $H, A$ be a strict subgroup of $G$, and $a, b$ be elements of $G$. Then $h(a) \cdot h(b) \cdot h^{\circ} A=$ $h^{\circ}(a \cdot b \cdot A)$ and $h^{\circ} A \cdot h(a) \cdot h(b)=h^{\circ}(A \cdot a \cdot b)$.

(25) Let $G, H$ be strict groups, $h$ be a homomorphism from $G$ to $H, A$ be a strict subgroup of $G, a, b$ be elements of $G, H_{1}$ be a subgroup of $\operatorname{Im} h$, and $a_{1}, b_{1}$ be elements of $\operatorname{Im} h$. If $a_{1}=h(a)$ and $b_{1}=h(b)$ and $H_{1}=h^{\circ} A$, then $a_{1} \cdot b_{1} \cdot H_{1}=h(a) \cdot h(b) \cdot h^{\circ} A$.

(26) Let $G, H$ be strict groups, $h$ be a homomorphism from $G$ to $H, G_{1}$ be a strict subgroup of $G, G_{2}$ be a strict normal subgroup of $G, H_{1}$ be a strict subgroup of $\operatorname{Im} h$, and $H_{2}$ be a strict normal subgroup of $\operatorname{Im} h$. Suppose

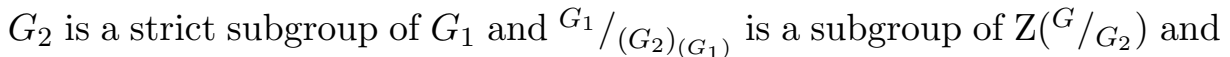
$H_{1}=h^{\circ} G_{1}$ and $H_{2}=h^{\circ} G_{2}$. Then $H_{1} /\left(H_{2}\right)_{\left(H_{1}\right)}$ is a subgroup of $\mathrm{Z}\left(\operatorname{Im} h / H_{2}\right)$.

(27) Let $G, H$ be strict groups, $h$ be a homomorphism from $G$ to $H$, and $A$ be a strict normal subgroup of $G$. Then $h^{\circ} A$ is a strict normal subgroup of $\operatorname{Im} h$.

Let $G$ be a strict nilpotent group, let $H$ be a strict group, and let $h$ be a homomorphism from $G$ to $H$. One can check that $\operatorname{Im} h$ is nilpotent.

Let $G$ be a strict nilpotent group and let $N$ be a strict normal subgroup of $G$. Note that $G / N$ is nilpotent.

One can prove the following three propositions:

(28) Let $G$ be a group. Given a finite sequence $F$ of elements of the normal subgroups of $G$ such that

(i) $\quad$ len $F>0$,

(ii) $F(1)=\Omega_{G}$,

(iii) $F($ len $F)=\{\mathbf{1}\}_{G}$, and 
(iv) for every $i$ such that $i, i+1 \in \operatorname{dom} F$ and for every strict normal subgroup $G_{1}$ of $G$ such that $G_{1}=F(i)$ holds $\left[G_{1}, \Omega_{G}\right]=F(i+1)$.

Then $G$ is nilpotent.

(29) Let $G$ be a group. Given a finite sequence $F$ of elements of the normal subgroups of $G$ such that

(i) $\quad$ len $F>0$,

(ii) $F(1)=\Omega_{G}$,

(iii) $F($ len $F)=\{\mathbf{1}\}_{G}$, and

(iv) for every $i$ such that $i, i+1 \in \operatorname{dom} F$ and for all strict normal subgroups $G_{1}, G_{2}$ of $G$ such that $G_{1}=F(i)$ and $G_{2}=F(i+1)$ holds $G_{2}$ is a subgroup of $G_{1}$ and ${ }^{G} / G_{2}$ is a commutative group.

Then $G$ is nilpotent.

(30) Let $G$ be a group. Given a finite sequence $F$ of elements of the normal subgroups of $G$ such that

(i) len $F>0$,

(ii) $F(1)=\Omega_{G}$,

(iii) $F($ len $F)=\{\mathbf{1}\}_{G}$, and

(iv) for every $i$ such that $i, i+1 \in \operatorname{dom} F$ and for all strict normal subgroups $G_{1}, G_{2}$ of $G$ such that $G_{1}=F(i)$ and $G_{2}=F(i+1)$ holds $G_{2}$ is a subgroup of $G_{1}$ and ${ }^{G} / G_{2}$ is a cyclic group.

Then $G$ is nilpotent.

Let us mention that every group which is nilpotent is also solvable.

\section{REFERENCES}

[1] Grzegorz Bancerek. The fundamental properties of natural numbers. Formalized Mathematics, 1(1):41-46, 1990.

[2] Grzegorz Bancerek and Krzysztof Hryniewiecki. Segments of natural numbers and finite sequences. Formalized Mathematics, 1(1):107-114, 1990.

[3] Czesław Byliński. Functions and their basic properties. Formalized Mathematics, 1(1):5565, 1990.

[4] Czesław Byliński. Functions from a set to a set. Formalized Mathematics, 1(1):153-164, 1990.

[5] Wojciech A. Trybulec. Classes of conjugation. Normal subgroups. Formalized Mathematics, 1(5):955-962, 1990.

[6] Wojciech A. Trybulec. Groups. Formalized Mathematics, 1(5):821-827, 1990.

[7] Wojciech A. Trybulec. Subgroup and cosets of subgroups. Formalized Mathematics, $1(\mathbf{5}): 855-864,1990$.

[8] Wojciech A. Trybulec. Commutator and center of a group. Formalized Mathematics, 2(4):461-466, 1991.

[9] Wojciech A. Trybulec and Michał J. Trybulec. Homomorphisms and isomorphisms of groups. Quotient group. Formalized Mathematics, 2(4):573-578, 1991.

[10] Katarzyna Zawadzka. Solvable groups. Formalized Mathematics, 5(1):145-147, 1996.

Received November 10, 2009 